(TNF $\alpha$ ) and interleukin 1 (IL1) have a critical role in the pathogenesis of RA. Biological treatment blocks pathological pathways in the actions of these proinflammatory cytokines. The aim of this study was to analyse concentrations of TNF $\alpha$ in blood serum and synovial fluid in patients with RA to identify patient groups most suitable for anti-TNF $\alpha$ treatment.

Methods Blood serum and synovial fluid from patients with RA and osteoarthritis (OA) at Pauls Stradins Clinical University Hospital Center of Rheumatology were selected for detection of TNF $\alpha$ from January 2005 to December 2007 by ELISA. Histiocyte/macrophage counts were detected in synovial fluid in the hospital laboratory. Statistical analyses were performed by the Student $t$ test.

Results 29 patients with RA and 11 with OA participated in the study; 17 of the patients with RA had high disease activity (DAS >5.1) and 12 had medium disease activity (DAS $\leq 1)$. A statistically significant difference was detected in TNF $\alpha$ levels in the synovial fluid of the RA group and the OA group $(p=019)$, and in serum from the RA group versus the OA group $(p=000154)$; medium activity RA group vs OA group TNF $\alpha$ in blood serum and synovial fluid $(p=007$ and $p=02)$ and high activity RA group vs OA group TNF $\alpha$ in blood serum and synovial fluid $(p<0.001$ and $p=001)$. The difference between high and medium RA activity groups in TNF $\alpha$ levels in serum and synovial fluid was $p=162$ and $p=0037$. Histiocyte/macrophage count in synovial fluid differed in OA group vs RA medium disease activity group $(p=02)$ and in OA vs RA high disease activity group ( $p=004)$, but in medium disease activity RA vs high disease activity RA group $(p=58)$.

Conclusion Further studies are needed to detect cytokines in blood serum and synovial fluid for the use of one as a possible marker when choosing treatment for individual patients with RA.

\section{A100 TUMOUR NECROSIS FACTOR $\alpha$ IN RHEUMATOID ARTHRITIS AND OSTEOARTHRITIS PATIENTS IN BLOOD SERUM AND SYNOVIAL FLUID}

I Bulina, ${ }^{1,2}$ D Andersone, ${ }^{1,2} \mathrm{~A}$ Sochnev, ${ }^{4,5}$ V Lavrentjevs, ${ }^{2} \mathrm{~J}$ Arajs, ${ }^{2}$ I Astica, ${ }^{2}$ E Sikora, ${ }^{2}$ J Zepa, ${ }^{2}$ I Jaunalksne, ${ }^{3}$ L Kovalchuk ${ }^{3,5}{ }^{1}$ Latvian University, ${ }^{2}$ Center of Rheumatology, Pauls Stradins Clinical University Hospital, Riga, Latvia ${ }^{3}$ Clinic of Clinical Immunology, Pauls Stradins Clinical University Hospital, Riga, Latvia ${ }^{4}$ Riga Stradins University, Riga, Latvia ${ }^{5}$ Riga Stradins University Institute of Immunology, Riga, Latvia

10.1136/ard.2010.129627m

Objective Rheumatoid arthritis (RA) is a chronic autoimmune disease of unknown cause which affects the ability of elderly people to work. There is strong evidence to suggest that inflammatory mediators such as tumour necrosis factor $\alpha$ 